\title{
TEM Characterization of a Refractory HEA Synthesized by High Energy Milling
}

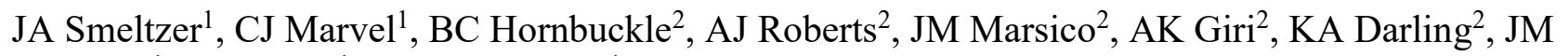 \\ Rickman $^{1}$, HM Chan ${ }^{1}$ and MP Harmer ${ }^{1}$ \\ 1. Department of Materials Science and Engineering, Lehigh University, Bethlehem, PA, USA. \\ 2. Weapons and Materials Research Directorate, U.S. Army Research Laboratory, Aberdeen Proving \\ Ground, MD, USA.
}

High entropy alloys (HEAs) are a new class of materials that have attracted intense research interest worldwide [1]. Unlike conventional alloys, they generally consist of multiple ( $>5$ ) principal components in equimolar or near equimolar ratios. The identification of new solid solution phases in these systems has been attributed to the increased configurational entropy, hence their designation as HEAs. This concept has opened an expansive realm of unexplored alloy compositions and microstructures. Taking into consideration the number of metallic elements in the periodic table, the number of nearly-equimolar HEAs with 5 or more components is in the millions! Although a large proportion of HEAs are single phase solid solutions, many also exhibit complex multi-phase microstructures. To date, the majority of studies have focused on HEAs with constituent elements derived from the group of 3-d transition metal elements; so-called refractory HEAs with constituent elements such as Mo, Nb, Ta, V, W, Ti, Zr, Hf, V, and Ta, have received less attention [2]. However, the unique mechanical/thermal properties observed in some of these materials have captured the imagination of the scientific community. For example, in terms of high temperature behavior, it was shown by Senkov et al. that $\mathrm{V}_{20} \mathrm{Nb}_{20} \mathrm{Mo}_{20} \mathrm{Ta}_{20} \mathrm{~W}_{20}$ outperforms industry standards Inconel 718 and Haynes 230, retaining high strength up to $1600{ }^{\circ} \mathrm{C}$ [3].

Most of the prior work on refractory HEAs has been carried out on alloys prepared by arc-melting. Because as cast structures tend to exhibit inter-dendritic segregation, thermomechanical treatments may be necessary to achieve a uniform microstructure. The aim of the current study was to explore the effect of a lesser studied fabrication technique, namely high energy milling, also referred to as mechanical alloying. Starting from high purity $(99.9 \%)$ elemental powders, a refractory HEA was prepared by highenergy cryogenic mechanical alloying using a modified SPEX 8000M shaker mill. The nominal composition of the HEA was $\mathrm{Nb}_{25} \mathrm{Mo}_{25} \mathrm{Ta}_{25} \mathrm{~W}_{25}$. The milling vials were back-filled with Ar and liquid $\mathrm{N}_{2}$ was used to maintain a milling temperature of $-196{ }^{\circ} \mathrm{C}$, and the milling media was $440 \mathrm{C}$ tool steel. No process control agents were used in the milling process. The powders were subsequently compacted by uniaxial pressing.

Thin specimens were prepared using in-situ FIB techniques and aberration-corrected TEM characterization (JEOL ARM-200CF, $200 \mathrm{kV}$ ) was carried out on consolidated powder samples both in the as-milled condition and after annealing for $1000 \mathrm{~h}$ at $1200{ }^{\circ} \mathrm{C}$ in argon. As evidenced by electron diffraction, the as-milled samples were mostly amorphous with small unmixed particles of W. However, STEM-HAADF imaging did reveal some isolated nanocrystalline grains that were 5-10 nm in diameter. The microstructures of the as-milled and annealed HEA samples are shown in Figure 1. It can be seen that recrystallization of the HEA has taken place, resulting in an equiaxed fine grain structure with grain diameters on average of approximately $200 \mathrm{~nm}$. It was also observed that the grain boundaries were decorated with particulate-like features. These exhibited dark intensities under HAADF imaging conditions, potentially indicating a lower average atomic number relative to the matrix grains. Compositional analysis of these features was carried out using high-resolution EDS, and it was 
determined that the particulates were enriched in argon (see Figure 2). It was concluded, therefore, that the 'particles' were in fact bubbles of argon that had been incorporated in the HEA compact, either during the annealing process or during milling due to the Ar atmosphere in the milling vial. The incorporation of gas media during high energy milling is an established phenomenon [4], and notably, it is known that gas bubbles can inhibit grain growth and therefore stabilize the nanocrystalline material against grain growth [5]. The extent to which argon bubbles plays a similar role for $\mathrm{Nb}_{25} \mathrm{Mo}_{25} \mathrm{Ta}_{25} \mathrm{~W}_{25}$ would require further study. Finally, as exhibited by the differing grain contrast in Figure $1 \mathrm{~b}$ under HAADF imaging, the HEA alloy consists of several phases, and is not the single phase solid solution that would have been predicted for this composition [6,7].

\section{References:}

[1] DB Miracle and ON Senkov, Acta Mat. 122 (2017), p. 448.

[2] ON Senkov et al., J. Mater. Res. 33 (2018), p. 3092.

[3] ON Senkov et al., Intermetallics 19 (2011), p. 698

[4] Y Muramatsu et al., Materials Transactions 46 (2005), p. 681

[5] MS Veshchunov, Materials (Basel) 2 (2009), p. 1252.

[6] X Yang and Y Zhang, Mater. Chem. Phys. 132 (2012), p. 233

[7] The support of ONR under grant \# N00014-18-1-2181, monitored by D. Shifler, is gratefully acknowledged.
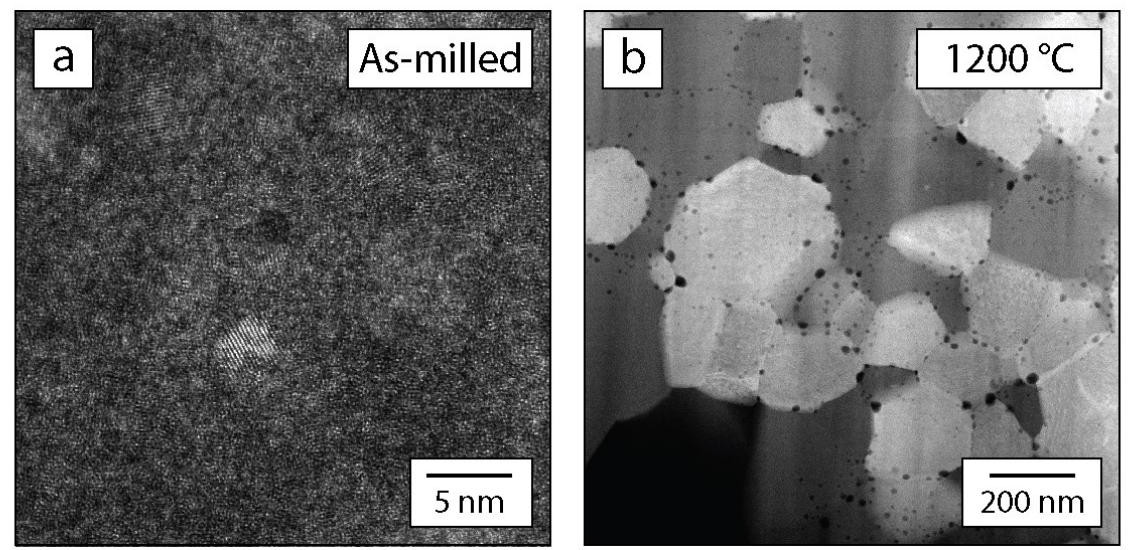

Figure 1. STEM-HAADF micrographs of the HEA: (a) as-milled and (b) annealed at $1200{ }^{\circ} \mathrm{C}$ for 100 hours.
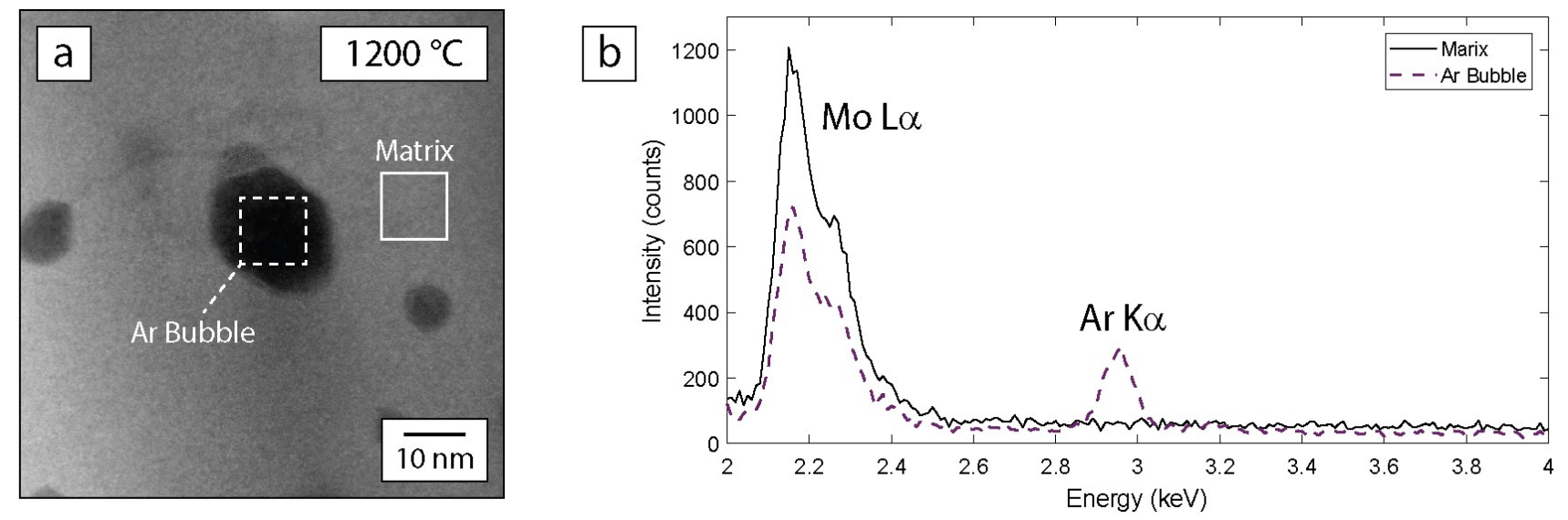

Figure 2. EDS analysis of an Ar Bubble including (a) STEM-HAADF reference image and (b) EDS spectra. 